\title{
Distributed Multiobjective Optimization for Network Resource Allocation of Multiagent Systems
}

Link to publication record in Manchester Research Explorer

\section{Citation for published version (APA):}

Li, Z., \& Ding, Z. (2020). Distributed Multiobjective Optimization for Network Resource Allocation of Multiagent Systems. IEEE Transactions on Cybernetics. https://ieeexplore.ieee.org/abstract/document/8955922

\section{Published in:}

IEEE Transactions on Cybernetics

\section{Citing this paper}

Please note that where the full-text provided on Manchester Research Explorer is the Author Accepted Manuscript or Proof version this may differ from the final Published version. If citing, it is advised that you check and use the publisher's definitive version.

\section{General rights}

Copyright and moral rights for the publications made accessible in the Research Explorer are retained by the authors and/or other copyright owners and it is a condition of accessing publications that users recognise and abide by the legal requirements associated with these rights.

\section{Takedown policy}

If you believe that this document breaches copyright please refer to the University of Manchester's Takedown Procedures [http://man.ac.uk/04Y6Bo] or contact uml.scholarlycommunications@manchester.ac.uk providing relevant details, so we can investigate your claim.

\section{OPEN ACCESS}




\title{
Distributed Multiobjective Optimization for Network Resource Allocation of Multiagent Systems
}

\author{
Zhongguo Li ${ }^{\circledR}$, Student Member, IEEE, and Zhengtao Ding ${ }^{\circledR}$, Senior Member, IEEE
}

\begin{abstract}
In this article, a distributed multiobjective optimization problem is formulated for the resource allocation of network-connected multiagent systems. The framework encompasses a group of distributed decision makers in the subagents, where each of them possesses a local preference index. Novel distributed algorithms are proposed to solve such a problem in a distributed manner. The weighted $L_{p}$ preference index is utilized in each agent since it can provide a robust Pareto solution to the problem. By using distributed fixed-time optimization methods, the $L_{p}$ preference index is constructed online without specifying the unknown parameters. Then, it is proved that the problem admits a unique Pareto solution. By exploiting consensus and gradient descent techniques, asymptotic convergence to the optimal solution is established via Lyapunov theories. Distinct from most of the current works, the proposed framework does not require any prior information in the formulation process, and private data can be well protected using this distributed approach. Numerical examples are included to validate the effectiveness of the proposed algorithms.
\end{abstract}

Index Terms-Distributed algorithms, multiagent systems, multiobjective optimization, resource allocation.

\section{INTRODUCTION}

D UE TO urgent needs in optimization of large-scale networks and recent advances in control of multiagent systems, distributed optimization problems have received considerable research attention during the past decade [1]-[4]. Among these problems, optimal resource allocation is regarded as one of the most important subjects due to its wide applications in many engineering fields, such as optimal power resource management [5], wireless communication resource allocation [6], and traffic-flow control problems [7].

Recently, studies on resource allocation mostly concentrate on single-objective problems (see [8]-[10]). Noticeably, in many applications, there inherently exist multiple conflicting objectives. For instance, economic, environmental, and technical objectives should be included simultaneously in a resource allocation problem of power systems [11]. One of the widely used methods for multiobjective optimization problems

Manuscript received May 29, 2019; revised September 12, 2019 and November 29, 2019; accepted December 9, 2019. This work was supported by the Science and Technology Facilities Council through Newton Fund under Grant ST/N006852/1. This article was recommended by Associate Editor J. Chen. (Corresponding author: Zhengtao Ding.)

The authors are with the Department of Electrical and Electronic Engineering, University of Manchester, Manchester M13 9PL, U.K. (e-mail: zhongguo.li@manchester.ac.uk; zhengtao.ding@manchester.ac.uk).

Color versions of one or more of the figures in this article are available online at http://ieeexplore.ieee.org.

Digital Object Identifier 10.1109/TCYB.2019.2961475
(MOPs) is the scalarization approach, for example, [12], which converts multiple objectives to a single weighted sum, and then solves it with traditional optimization methods. Although this approach holds high computational efficiency and many welldeveloped tools for single-objective optimization are available, it requires some prior knowledge to choose the weighting factors. Currently, many other techniques, such as particle swarm optimization [13] and evolutionary approaches [14]-[17] are extensively studied. In [16] and [17], MOPs are decomposed into a set of scalar subproblems. The algorithm in [16] uses a constrained decomposition with grids to achieve both better robustness to the shape of the Pareto front and high solution diversity. A constraint-based method is proposed in [17], which selects one of the objectives as the main objective and takes the others as constraints.

The aforementioned works are mainly based on centralized algorithms where all objective functions need to be collected and combined in advance for centralized computation. A large amount of results in literature have been contributed to multiobjective optimization algorithms in a centralized manner, but most of them are evolutionary algorithms, as in [14], [15], and [18], by which only near-Pareto optimum can be obtained with stochastic convergence properties. In addition, evolutionary algorithms usually require significant computation resources and massive data communication. Even for single-objective optimization problems, the centralized approaches have struggled with the computation and communication complexity. More recently, distributed methods are widely considered as potential substitutes to the centralized ones, since they have a number of superior advantages, such as fast parallel computation, privacy protection, and robustness to single endpoint failures [19]. Since adding more objectives will dramatically compound the problems, and in many real-world applications, the participants are usually not willing to share their private information, for example, local cost functions and preferences, the aforementioned benefits of distributed methods are of great importance to multiobjective resource allocation problems (MRAPs).

In [20], a distributed algorithm is proposed using a weighted sum approach to search the Pareto front of an MOP without global constraints. One limitation of the study is that the weighting parameters are chosen in advance as global information, in which sense the algorithm is not fully distributed. The work [21] addresses MOPs with gradient noises via diffusion strategies, where stochastic convergence to the Pareto solution with bounded error is studied. Both [20] and [21] consider the cases where each agent is only assigned 
with one single objective, and use the weighted sum approach to integrate the objectives. The weighted $L_{p}$ preference-based method is investigated in [22], where comparisons among different settings of the value $p$ are conducted in terms of the robustness and selective pressure.

Algorithms without prior knowledge and high-level decision-making processes are urgently needed for MRAPs to achieve fully autonomous and mechanized operations. In addition, to facilitate the requirements of distributed multiagent networks, the algorithms should be decentralized into a group of segments with local communications. However, theoretical progress on such problems is lagging far behind. There are a series of challenges that impede the algorithm development. First, selections of preference indices that suitably compromise among the objectives are difficult [23]. Second, the design of distributed algorithms for single-objective resource allocation problems remains a severe issue due to the complexity of convergence analysis, and challenges in handling global constraints [8]. Especially, dealing with the combination of them is nontrivial, because some difficulties will arise, such as adjusting the preference index during optimization processes in a distributed manner, and analyzing convergence properties of the compound problems.

Motivated by the above observations, this article aims to establish a framework for MRAPs and proposes distributed algorithms to seek the most satisfactory Pareto solution. The problems under consideration are of fundamental differences with respect to the state-of-the-art works [16], [17] as discussed above. By allocating each subagent a local decision maker, we decentralize the problem into a group of subagents communicating over a network. In particular, a weighted $L_{p}$ preference index is introduced to quantify the Pareto solutions, where two unknown parameters need to be acquired as the optimization proceeds. Such an $L_{p}$ preference index proves to be effective in producing robust Pareto optimum for practical applications, as the resulting solution is close to the ideal vector. In order to formulate the preference index, we exploit two fixed-time optimization algorithms in a distributed manner, by which the agents can quickly obtain their local preference indices within a fixed time. The convergence of those algorithms is established by exploring the graph properties and fixed-time control techniques. Although decision makers are involved, their preference indices are learned from the optimization process utilizing auxiliary dynamics without prior information. Due to the basic requirement of eliminating global information in distributed algorithms, the weighting vector for each type of objective is also assumed to be unknown, that is, the network objectives can be any combination of the distributed local ones. This is different from the single-objective optimization problems in [2], [3], and [8] that assume the network objective is the summation of all local objectives. With some commonly used assumptions, it is proved that the proposed framework admits a unique Pareto solution such that the preference metrics of the group are minimized. Then, distributed algorithms for constrained optimization problems are proposed to search for the optimal decisions, and deterministic convergence is established via applying the Lyapunov theory, consensus techniques, and convex analysis. To date, none of the existing works deal with the problem where each local agent maintains multiple conflicting objectives for large-scale distributed multiagent systems. The significance of our research is to provide a new solution for distributed MRAPs that does not require any weighting parameters. In particular, such distributed algorithms can be implemented online using a set of low-price processors with potentially less communication and computation resources. Numerical simulations are performed to validate the effectiveness of the proposed algorithms, where comparisons with respect to the centralized methods and the scalability studies are carried out.

The remainder of this article is organized as follows. Some preliminaries on graph theory and formulation of MRAPs are presented in Section II. Distributed algorithms and convergence analysis are studied in Section III. Simulation examples are included for illustration in Section IV. Section V concludes this article.

Notations: Throughout this article, we denote $\mathbb{R}, \mathbb{R}_{\geq 0}$, $\mathbb{R}_{>0}, \quad \mathbb{R}^{n}$, and $\mathbb{R}^{n \times m}$ as the sets of real numbers, nonnegative real numbers, positive real numbers, $n$-dimensional real vectors, and real matrices of size $n \times m$, respectively. Let $\operatorname{diag}\left(d_{1}, d_{2}, \ldots, d_{n}\right)$ be a diagonal matrix with elements $\left(d_{1}, d_{2}, \ldots, d_{n}\right)$ on its main diagonal. For $x \in \mathbb{R}^{n}$, the gradient of a differentiable function $F(x): \mathbb{R}^{n} \rightarrow \mathbb{R}$ with respect to $x_{i}$ is denoted by $\nabla_{i} F(x)$, and the gradient with respect to $x$ is represented by $\nabla F(x)=\left[\nabla_{1} F(x), \ldots, \nabla_{n} F(x)\right]^{T}$.

\section{PReliminaries AND Problem Formulation}

\section{A. Graph Theory}

A graph is denoted by $\mathcal{G}(\mathcal{V}, \mathcal{E})$, where $\mathcal{V}=\{1,2, \ldots, N\}$ represents a set of $N$ distinct vertices, denoting the participants in the network, and $\mathcal{E}=\{(i, j): i, j \in \mathcal{V}\}$ denotes the set of edges, representing the communication channels among the participants. If any edge pair $(i, j) \in \mathcal{E}$ implies $(j, i) \in \mathcal{E}$, then the graph is said to be undirected. The elements of the adjacency matrix, denoted as $\mathcal{A}$, are defined as $a_{i j}=1$ if the edge pair $(j, i) \in \mathcal{E}$, and zero otherwise. We define the neighboring set of the $i$ th agent as $\mathcal{N}_{i}=\{j \in \mathcal{V}:(j, i) \in \mathcal{E}\}$, by which the degree matrix is defined as $\mathcal{D}=\operatorname{diag}\left(d_{1}, d_{2}, \ldots, d_{n}\right)$, where $d_{i}=\sum_{j=1}^{N} a_{i j} \forall i \in \mathcal{V}$. The Laplacian matrix is given by $\mathcal{L}=\mathcal{D}-\mathcal{A}$. A graph $\mathcal{G}(\mathcal{V}, \mathcal{E})$ is said to be connected if there exists a path from one vertex to any others. For an undirected and connected graph, 0 is a simple eigenvalue of the Laplacian matrix $\mathcal{L}$, and all other eigenvalues are positive [24].

\section{B. Preliminary Lemmas}

Lemma 1 [25]: Let $x_{i} \geq 0$ for all $i=1, \ldots, L$. If $0<p \leq 1$, then

$$
\sum_{i=1}^{L} x_{i}^{p} \geq\left(\sum_{i=1}^{L} x_{i}\right)^{p}
$$

If $p>1$

$$
\sum_{i=1}^{L} x_{i}^{p} \geq L^{1-p}\left(\sum_{i=1}^{L} x_{i}\right)^{p}
$$




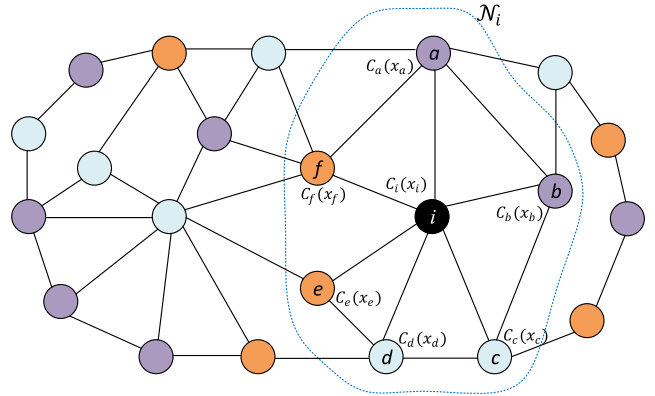

Fig. 1. Network structure of a resource allocation problem. Each node denotes a distributed agent, where different colors represent that the cost functions or preferences of the participants are different, and the line segments denote the communication network.

Lemma 2 [26]: Consider a continuous and radially unbounded function $V(x(t)): \mathbb{R}^{m} \rightarrow \mathbb{R}_{\geq 0}$, satisfying

$$
\dot{V}(x(t)) \leq-\left[\alpha V^{\mu}(x(t))+\beta V^{\nu}(x(t))\right]^{\rho}
$$

where $\alpha, \beta, \rho, \mu, \nu \in \mathbb{R}_{>0}$ with $\rho \mu<1$ and $\rho \nu>1$. Then, the origin is a fixed-time stable equilibrium, and the upper bound of the settling time is given by

$$
T=\frac{1}{\alpha^{\rho}(1-\rho \mu)}+\frac{1}{\beta^{\rho}(\rho v-1)} .
$$

\section{Problem Formulation}

Consider a group of $N$ agents that cooperatively optimize some objective functions, where the $i$ th agent maintains a set of $K$ conflicting or partially conflicting objectives

$$
C_{i}\left(x_{i}\right)=\left\{C_{i}^{1}\left(x_{i}\right), C_{i}^{2}\left(x_{i}\right), \ldots, C_{i}^{K}\left(x_{i}\right)\right\}
$$

where $C_{i}^{k}\left(x_{i}\right): \mathbb{R} \rightarrow \mathbb{R}$ denotes the $k$ th objective of agent $i$, and is privately known to agent $i$ only. The network resource constraint is given by

$$
\sum_{i=1}^{N} x_{i}=\sum_{i=1}^{N} d_{i}
$$

For a nonconflicting multiobjective problem, a simple solution that simultaneously optimizes all objectives can be obtained, and it can therefore be converted to single-objective optimization problems. To avoid this trivial case, the objectives are assumed to be at least partially conflicting, which means a compromised solution preferred by the decision makers should be derived. Fig. 1 illustrates a network structure of MRAPs, where each node represents a participant that possesses multiple conflicting objectives, and the line segments denote the communication channels among the participants. Under distributed settings, it is assumed that agent $i$ can only communicate with its adjacent neighbors $\mathcal{N}_{i}$ (encircled by the dotted line) to share some nonconfidential information. Moreover, all participants are subject to the network resource constraint in (6).

The following assumptions are made on the local objective functions and the communication topology.
Assumption 1: Each local objective function $C_{i}^{k}$ is twice differentiable, and $m_{i}^{k}$-strongly convex with $m_{i}^{k}>0$, that is, there exists a positive $m_{i}^{k}$ such that

$$
\begin{gathered}
\left(\nabla C_{i}^{k}\left(x_{i}\right)-\nabla C_{i}^{k}\left(x_{i}^{\prime}\right)\right)^{T}\left(x_{i}-x_{i}^{\prime}\right) \\
\geq m_{i}^{k}\left\|x_{i}-x_{i}^{\prime}\right\|^{2} \forall x_{i}, x_{i}^{\prime} \in \mathbb{R} .
\end{gathered}
$$

Assumption 2: The communication network is represented by an undirected and connected graph.

To facilitate the ensuing analysis, let the objectives of the network be $\mathbb{C}=\left\{\mathbb{C}^{1}(x), \ldots, \mathbb{C}^{K}(x)\right\}$, where $x=$ $\left[x_{1}, \ldots, x_{N}\right]^{T} \in \mathbb{R}^{N}$, and $\mathbb{C}^{k}(x)=\sum_{i=1}^{N} \varpi_{i}^{k} C_{i}^{k}$ is the $k$ th objective of the network, with $\varpi^{k}=\left[\varpi_{1}^{k}, \ldots, \varpi_{N}^{k}\right]^{T} \in \mathbb{R}_{>0}^{N}$ being some weighting vector relying on the preference metrics.

Remark 1: Such a weighting vector $\varpi^{k}$ always exists, due to the convexity of the problem [27]. The relationship between local objectives and the network objective is usually considered as $\sum_{i=1}^{N} C_{i}\left(x_{i}\right)$ in single-objective optimization problems [3], [8], while in this article, the weight $\varpi^{k}$ is determined by the decision makers and is assumed to be unknown. Here, introducing the weighting vector is for analytical purposes only, which is not required in the proposed algorithms.

Pareto optimality is a widely used concept for MOPs. Now, we give the formal definition as follows.

Definition 1 (Pareto Optimality [23]): A decision vector $x^{*} \in \mathbb{R}^{N}$ is a Pareto optimum of the MRAP if there does not exist any other decision vector $x \in \mathbb{R}^{N}$ satisfying the constraint (6) such that $\mathbb{C}^{k}(x) \leq \mathbb{C}^{k}\left(x^{*}\right)$, for all $k=1, \ldots, K$ with $\mathbb{C}^{j}(x)<\mathbb{C}^{j}\left(x^{*}\right)$ for at least one index $j$.

In this article, the global decision-making preference index is given by

$$
\begin{aligned}
U\left(x, \hat{x}^{*}, \omega^{*}\right) & =\sum_{i=1}^{N}\left[\sum_{k=1}^{K} \omega_{i}^{k *}\left(C_{i}^{k}\left(x_{i}\right)-C_{i}^{k}\left(\hat{x}_{i}^{*}\right)\right)^{p}\right]^{\frac{1}{p}} \\
1 & \leq p<\infty
\end{aligned}
$$

where $C_{i}^{1}\left(\hat{x}_{i}^{1 *}\right), C_{i}^{2}\left(\hat{x}_{i}^{2 *}\right), \ldots, C_{i}^{K}\left(\hat{x}_{i}^{K *}\right)$ denote the ideal points of each objective of the $i$ th agent; $\hat{x}^{*}=\operatorname{col}\left(\hat{x}_{1}^{*}, \ldots, \hat{x}_{N}^{*}\right) \in \mathbb{R}^{K N}$ is the ideal decision variable of the network, with $\hat{x}_{i}^{*}=$ $\left[\hat{x}_{i}^{1 *}, \ldots, \hat{x}_{i}^{K *}\right]^{T} \in \mathbb{R}^{K}$ being the ideal decision variable of agent $i ; \omega^{*}=\operatorname{col}\left(\omega_{1}^{*}, \ldots, \omega_{N}^{*}\right) \in \mathbb{R}^{K N}$ are positive weightings of the objectives specified by the local decision makers, with $\sum_{k=1}^{K} \omega_{i}^{k *}=1$ for all $i \in \mathcal{V}$. As in [23], the weight $\omega_{i}^{k *}$ is chosen according to the relative importance of objective $C_{i}^{k}$ compared with the total objective value $C_{i}$

$$
\omega_{i}^{k *}=\frac{\left|C_{i}^{k}\left(\bar{x}_{i}^{k *}\right)\right|}{\sum_{j=1}^{K}\left|C_{i}^{j}\left(\bar{x}_{i}^{*}\right)\right|}
$$

where $\bar{x}_{i}^{k *}$ denotes the optimizer for the summation of each type objective function subject to the resource constraint, that is, for all $k=1, \ldots, K$ and $i \in \mathcal{V}$

$$
\bar{x}_{i}^{k *}=\underset{\bar{x}_{i}^{k} \in \mathbb{R}}{\operatorname{argmin}} \sum_{i=1}^{N} C_{i}^{k}\left(\bar{x}_{i}^{k}\right), \text { subject to (6). }
$$


Remark 2: This class of preference index in (7) is called the weighted $L_{p}$ metrics that have been commonly used to generate Pareto-optimal solutions in a lot of literature, for instance, [28] and [29]. It is worth noting that the preference index (7) contains two unknown parameters $\omega_{i}^{k *}$ and $C_{i}^{k}\left(\hat{x}_{i}^{*}\right)$, which will be learned from the optimization process. In some existing works, for example, [20] and [27], the weight of each objective is selected randomly or chosen by a global agent with some prior information. However, prior knowledge and global information are usually not available for distributed optimization problems.

Remark 3: The problem (9) is a distributed form of singleobjective optimization. This article utilizes such a formulation to derive one of the parameters for the preferences. From the practical point of view, it has been observed that such weightings in (8) prove to be effective for many applications [30], which will also be demonstrated by the simulation examples in Section IV. Mathematically, the Pareto solution obtained from weighted $L_{p}$ metrics is robust to modeling uncertainties since it is unlikely to provide those solutions that are too far from the ideal points [28]. Nevertheless, this approach poses a significant challenge for distributed methods, because it requires that all agents cooperatively find the cost of each type of objective, that is, the summation of the $k$ th objective subject to the global constraint as in (9). To handle such a difficulty, we propose fixed-time algorithms to solve the distributed-constrained problem in Section III.

Conventionally, this MRAP can be solved via centralized approaches, such as multiobjective evolutionary algorithms, genetic algorithms, and particle swarm optimization [14]. For those centralized methods, all information of subsystems, including real-time data and objective functions, should be collected to a central unit (with data collection, calculation, and decision-making processors), where communication requirements are highly demanding, and private information may not be well protected. In addition, some of those methods are computationally expensive, and difficult to code. Consequently, centralized methods are not feasible for large-scale MRAPs.

To eliminate the aforementioned disadvantages of centralized approaches, this article proposes distributed algorithms, by which the complex MRAP can be solved using a group of low-price processors with local communications. It is assumed that there is a local decision maker in each agent that makes compromises among the objectives. The centralized decisionmaking criteria (7) are allocated to a group of distributed decision makers, where the preference index of the $i$ th agent is given by

$$
\begin{aligned}
u_{i}\left(x_{i}, \hat{x}_{i}^{*}, \omega_{i}^{*}\right) & =\left[\sum_{k=1}^{K} \omega_{i}^{k *}\left(C_{i}^{k}\left(x_{i}\right)-C_{i}^{k}\left(\hat{x}_{i}^{*}\right)\right)^{p}\right]^{\frac{1}{p}} \\
1 & \leq p<\infty
\end{aligned}
$$

It is important that the agents are entitled to formulate its decision criterion since there is no central authority allowed to collect the private information.

To this end, the MRAP can be formulated as

$$
\min _{x_{i} \in \mathbb{R}}\left\{C_{i}^{1}\left(x_{i}\right), \ldots, C_{i}^{K}\left(x_{i}\right)\right\} \text {, for all } i \in \mathcal{V}
$$

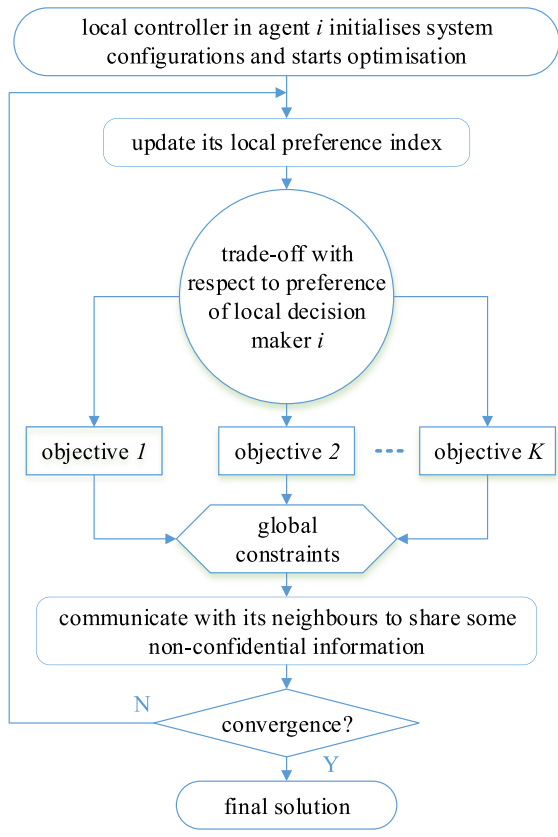

Fig. 2. Flowchart of multiobjective optimization for distributed agents. Each agent has its own preference used to compromise the local cost functions, and communicates with its adjacent neighbors to cooperatively optimize the network objectives.

$$
\text { subject to } \sum_{i=1}^{N} x_{i}=\sum_{i=1}^{N} d_{i}
$$

such that (10) is minimized.

Remark 4: Different from most of the recent research on multiobjective optimization that searches exhaustively for the complete Pareto set (usually referred to as vector optimization), this article considers the classic preferencebased methods using a distributed approach, which generates only one most satisfactory solution depending on the group's preferences. This class of preference-based methods is regarded as one of the most efficient approaches to deal with MOPs, and many studies have been devoted to the research area, for instance, [20]-[23] and [28]. Discussions and comparisons of the merits and demerits of the two classes of approaches have been well documented in [23] and [27].

Remark 5: The reformulated problem in (11) using the distributed preference index (10) is equivalent to the centralized one in (7) if the consensus of the Lagrangian multiplies is achieved. Detailed analysis has been well established in many existing works, for example, [5] and [9].

Fig. 2 displays a flowchart of distributed multiobjective optimization algorithms. For the local controller within the $i$ th agent, it initializes the system configurations and inputs some local information, including objective functions, initial decision preferences, and states. Then, agent $i$ compromises among its local objectives according to its decision maker. By communicating with adjacent neighbors, all agents will converge to the Pareto-optimal solution that satisfies the global constraint and mostly fits to the preferences specified by the decision-making group. It should be emphasized that the decision makers will amend their preferences during the optimization process. 


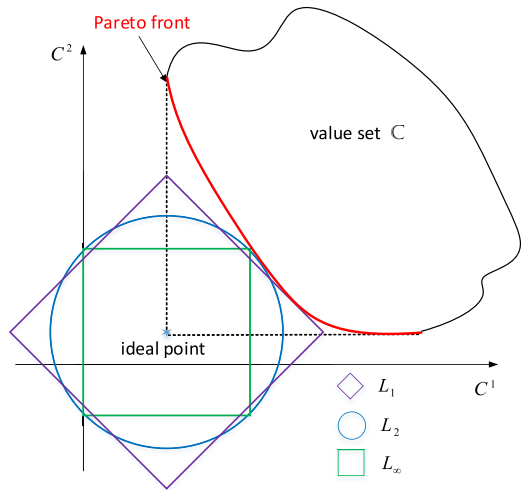

Fig. 3. Interpretation of unique Pareto optimum satisfying preference indices of decision makers. The preference indices are centered at the ideal point with $L_{p}$-norm distance.

\section{Distributed AlgorithmS AND CONVERGENCE ANALYSIS}

Before presenting the distributed algorithms, let us study some properties of problem (11).

Definition $2\left(\Lambda^{\geq}\right.$-Convex Set): A set $S \subset \mathbb{R}^{K}$ is defined as $\Lambda^{\geq}$-convex if and only if $S+\Lambda^{\geq}=\left\{s+d \mid s \in S, d \in \mathbb{R}_{\geq 0}^{K}\right\}$ is a convex set, with $\Lambda^{\geq}=\left\{d \in \mathbb{R}_{\geq 0}^{K}\right\}$ being the non-negative convex cone.

Lemma 3: Under Assumption 1, the set of the objective values of problem (11) is $\Lambda^{\geq}$-convex. Moreover, the proposed optimization problem admits a unique solution.

Proof: Notice that all of the objectives, $C_{i}^{j} \forall i \in \mathcal{V}, j=$ $1, \ldots, K$ are convex subject to a linear constraint. Thus, the MRAP is convex. Consider $f_{1}=\mathbb{C}\left(x_{1}\right)+d_{1}$ and $f_{2}=\mathbb{C}\left(x_{2}\right)+$ $d_{2}$, with $x_{1}, x_{2} \in \mathbb{R}^{N}$ and $d_{1}, d_{2} \in \Lambda^{\geq}$. Then, for any $\epsilon \in[0,1]$

$$
\begin{aligned}
\epsilon f_{1}+(1-\epsilon) f_{2}= & \epsilon \mathbb{C}\left(x_{1}\right)+(1-\epsilon) \mathbb{C}\left(x_{2}\right)+\epsilon d_{1}+(1-\epsilon) d_{2} \\
& \geq \mathbb{C}\left(\epsilon x_{1}+(1-\epsilon) x_{2}\right)+\epsilon d_{1}+(1-\epsilon) d_{2} \\
& \in \mathbb{C}+\Lambda^{\geq}
\end{aligned}
$$

where the inequality follows from the convexity of $\mathbb{C}$. Hence, the value set of the constrained problem (11) is $\Lambda^{\geq}$-convex, which implies the Pareto front of the solution set is convex. As a result, for any $1 \leq p<\infty$ in (10), the solution of the MRAP (11) is unique (see [28]).

To demonstrate the fundamental principle behind, an example with two objective functions is depicted in Fig. 3. For convex functions, from Lemma 3, the objective values constitute a $\Lambda^{\geq}$-convex set, with a convex Pareto front. By utilizing $L_{p}$ metrics for $1 \leq p<\infty$, there exists and only exists one unique solution satisfying the decision makers' preferences. Furthermore, it is evident that the solutions derived from $L_{p}$ metrics are usually close to the ideal point, as shown in Fig. 3, and therefore those solutions are robust to the model and parameter uncertainties in many engineering systems.

Remark 6: With Assumption 1, the Pareto front of problem (11) is guaranteed to be convex, which consequently ensures that all the Pareto solutions can be derived by varying the weighting parameters. This is crucial for the following algorithm design and convergence analysis. For nonconvex problems, some Pareto solutions may not be obtained by the weighted-preference-based approaches [28].
Next, we present the proposed algorithms. For each agent $i$, the constrained single-objective optimizer $\bar{x}_{i}^{k}$ and optimal weight $\omega_{i}^{k}$ are derived by

$$
\begin{aligned}
\dot{y}_{i}^{k}= & -\alpha\left[\sum_{j=1}^{N} a_{i j}\left(\nabla C_{j}^{k}\left(\bar{x}_{j}^{k}\right)-\nabla C_{i}^{k}\left(\bar{x}_{i}^{k}\right)\right)\right]^{\frac{m}{n}} \\
& -\beta\left[\sum_{j=1}^{N} a_{i j}\left(\nabla C_{j}^{k}\left(\bar{x}_{j}^{k}\right)-\nabla C_{i}^{k}\left(\bar{x}_{i}^{k}\right)\right)\right]^{2-\frac{m}{n}} \\
\bar{x}_{i}^{k}= & \sum_{j=1}^{N} a_{i j}\left(y_{j}^{k}-y_{i}^{k}\right)+\bar{x}_{i}^{k}(0) \\
\omega_{i}^{k}= & \frac{\left|C_{i}^{k}\left(\bar{x}_{i}^{k}\right)\right|}{\sum_{j=1}^{K}\left|C_{i}^{j}\left(\bar{x}_{i}^{j}\right)\right|} .
\end{aligned}
$$

The ideal point seeking algorithm is designed as

$$
\dot{\hat{x}}_{i}=-\gamma\left[\nabla C_{i}\left(\hat{x}_{i}\right)\right]^{\frac{m}{n}}-\delta\left[\nabla C_{i}\left(\hat{x}_{i}\right)\right]^{2-\frac{m}{n}} .
$$

Then, the compromised solution for agent $i$ can be obtained from

$$
\begin{aligned}
& \dot{x}_{i}=-\nabla u_{i}\left(x_{i}, \hat{x}_{i}, \omega_{i}\right)-\lambda_{i} \\
& \dot{\lambda}_{i}=-\sum_{j=1}^{N} a_{i j}\left(\lambda_{i}-\lambda_{j}\right)-\sum_{j=1}^{N} a_{i j}\left(z_{i}-z_{j}\right)+\left(x_{i}-d_{i}\right) \\
& \dot{z}_{i}=\sum_{j=1}^{N} a_{i j}\left(\lambda_{i}-\lambda_{j}\right) .
\end{aligned}
$$

In (12)-(14), $\alpha, \beta, \gamma$, and $\delta$ are positive real numbers; $m$ and $n$ are positive odd integers with $m<n$; and initial states satisfy $\sum_{i=1}^{N} \bar{x}_{i}^{k}(0)=\sum_{i=1}^{N} d_{i}$.

Now, we show the convergence analysis of the proposed algorithms (12)-(14). First, we prove that the weighting vectors of the preference indices converge to (8) within a fixed time.

Lemma 4: Under Assumptions 1 and 2, the proposed algorithm in (12) solves the constrained optimization problem (9). Moreover, the weighting parameters $\omega$ converge to $\omega^{*}$ in a fixed time, and the upper bound of settling time is given by

$$
\begin{aligned}
T_{\max }^{1}= & \frac{1}{\alpha 2^{\frac{3 n+m}{4 n}}\left(\frac{1}{2} \underline{\kappa}\left(\lambda_{2}(\mathcal{L})\right)^{2}\right)^{\frac{m+n}{2 n}}\left(1-\frac{3 n+m}{4 n}\right)} \\
& +\frac{1}{\beta 2^{\frac{5 n-m}{4 n}} N^{\frac{n-m}{2 n}}\left(\frac{1}{2} \underline{\kappa}\left(\lambda_{2}(\mathcal{L})\right)^{2}\right)^{\frac{3 n-m}{2 n}}\left(\frac{5 n-m}{4 n}-1\right)}
\end{aligned}
$$

where $\underline{\kappa}=\min _{i \in \mathcal{V}, k \in\{1, \ldots, K\}} m_{i}^{k}$, and $\lambda_{2}(\mathcal{L})$ denotes the second smallest eigenvalue of the Laplacian matrix.

Proof: Since the communication graph is undirected, we have

$$
\sum_{i=1}^{N} \bar{x}_{i}^{k}=\sum_{i=1}^{N} \sum_{j=1}^{N} a_{i j}\left(y_{j}^{k}-y_{i}^{k}\right)+\sum_{i=1}^{N} \bar{x}_{i}^{k}(0)=\sum_{i=1}^{N} d_{i}
$$


by which the equality constraint is guaranteed. Therefore, (12a)-(12c) can be transferred to an unconstrained optimization problem as

$$
\min _{y^{k} \in \mathbb{R}^{N}} C^{k}\left(y^{k}\right)=\sum_{i=1}^{N} C_{i}^{k}\left(\sum_{j=1}^{N} a_{i j}\left(y_{j}^{k}-y_{i}^{k}\right)+\bar{x}_{i}^{k}(0)\right)
$$

where $y^{k}=\left[y_{1}^{k}, \ldots, y_{N}^{k}\right]^{T}$. From (12b), we have

$$
\frac{\partial C_{i}^{k}}{\partial y_{j}^{k}}=\frac{\partial C_{i}^{k}}{\partial \bar{x}_{i}^{k}} \frac{\partial \bar{x}_{i}^{k}}{\partial y_{j}^{k}}= \begin{cases}-\sum_{j=1}^{N} a_{i j} \nabla C_{i}^{k}\left(\bar{x}_{i}^{k}\right), & j=i \\ a_{i j} \nabla C_{i}^{k}\left(\bar{x}_{i}^{k}\right), & j \neq i\end{cases}
$$

which implies

$$
\nabla C^{k}\left(y^{k}\right)=-\mathcal{L} \nabla C^{k}\left(\bar{x}^{k}\right) .
$$

Due to strong convexity of all $C_{i}^{k} \forall i \in \mathcal{V}, k=1, \ldots, K$, and connectivity of the graph, it follows that (see [31]):

$$
\left\|\nabla C^{k}\left(y^{k}\right)\right\|^{2} \geq \frac{1}{2} \underline{\kappa}\left(\lambda_{2}(\mathcal{L})\right)^{2}\left[C^{k}\left(y^{k}\right)-C^{k}\left(y^{k *}\right)\right]
$$

where $y^{k *}=\left[y_{1}^{k *}, \ldots, y_{N}^{k *}\right]^{T}$ is the optimal solution of the sum of the $k$ th objective subject to the equality constraint. Now, (12a) can be rewritten as

$$
\begin{aligned}
\dot{y}_{i}^{k}= & -\alpha\left(\sum_{j=1, j \neq i}^{N} \frac{\partial C_{j}^{k}}{\partial y_{i}^{k}}+\frac{\partial C_{i}^{k}}{\partial y_{i}^{k}}\right)^{\frac{m}{n}} \\
& -\beta\left(\sum_{j=1, j \neq i}^{N} \frac{\partial C_{j}^{k}}{\partial y_{i}^{k}}+\frac{\partial C_{i}^{k}}{\partial y_{i}^{k}}\right)^{2-\frac{m}{n}}
\end{aligned}
$$

and in a compact form as

$$
\dot{y}^{k}=-\alpha\left[\nabla C^{k}\left(y^{k}\right)\right]^{\frac{m}{n}}-\beta\left[\nabla C^{k}\left(y^{k}\right)\right]^{2-\frac{m}{n}} .
$$

Consider a Lyapunov candidate given by

$$
V_{1}=\frac{1}{2}\left[C^{k}\left(y^{k}\right)-C^{k}\left(y^{k *}\right)\right]^{2}
$$

of which the time derivative along (21) is obtained as

$$
\begin{aligned}
\dot{V}_{1}= & {\left[C^{k}\left(y^{k}\right)-C^{k}\left(y^{k *}\right)\right] \nabla^{T} C^{k}\left(y^{k}\right) } \\
& \times\left[-\alpha\left[\nabla C^{k}\left(y^{k}\right)\right]^{\frac{m}{n}}-\beta\left[\nabla C^{k}\left(y^{k}\right)\right]^{2-\frac{m}{n}}\right] \\
= & {\left[C^{k}\left(y^{k}\right)-C^{k}\left(y^{k *}\right)\right] } \\
& \times\left[-\alpha \sum_{i=1}^{N}\left(\frac{\partial C^{k}}{\partial y_{i}^{k}}\right)^{1+\frac{m}{n}}-\beta \sum_{i=1}^{N}\left(\frac{\partial C^{k}}{\partial y_{i}^{k}}\right)^{3-\frac{m}{n}}\right] .
\end{aligned}
$$

Applying Lemma 1, we have

$$
\begin{aligned}
\dot{V}_{1} \leq & -\alpha\left[C^{k}\left(y^{k}\right)-C^{k}\left(y^{k *}\right)\right]\left[\sum_{i=1}^{N}\left(\frac{\partial C^{k}}{\partial y_{i}^{k}}\right)^{2}\right]^{\frac{m+n}{2 n}} \\
& -\beta N^{\frac{n-m}{2 n}}\left[C^{k}\left(y^{k}\right)-C^{k}\left(y^{k *}\right)\right]\left[\sum_{i=1}^{N}\left(\frac{\partial C^{k}}{\partial y_{i}^{k}}\right)^{2}\right]^{\frac{3 n-m}{2 n}} .
\end{aligned}
$$

Substituting (19) into (25), and from (22), we can obtain

$$
\begin{aligned}
\dot{V}_{1} \leq & -\alpha\left(\frac{1}{2^{-}}\left(\lambda_{2}(\mathcal{L})\right)^{2}\right)^{\frac{m+n}{2 n}}\left(\left[C^{k}\left(y^{k}\right)-C^{k}\left(y^{k *}\right)\right]^{2}\right)^{\frac{3 n+m}{4 n}} \\
& -\beta N^{\frac{n-m}{2 n}}\left(\frac{1}{2^{-}} \underline{\kappa}\left(\lambda_{2}(\mathcal{L})\right)^{2}\right)^{\frac{3 n-m}{2 n}} \\
& \times\left(\left[C^{k}\left(y^{k}\right)-C^{k}\left(y^{k *}\right)\right]^{2}\right)^{\frac{5 n-m}{4 n}} \\
= & -\alpha 2^{\frac{3 n+m}{4 n}}\left(\frac{1}{2^{-}} \underline{\kappa}\left(\lambda_{2}(\mathcal{L})\right)^{2}\right)^{\frac{m+n}{2 n}}\left(V_{1}\right)^{\frac{3 n+m}{4 n}} \\
& -\beta 2^{\frac{5 n-m}{4 n}} N^{\frac{n-m}{2 n}}\left(\frac{1}{2^{-}} \underline{\kappa}\left(\lambda_{2}(\mathcal{L})\right)^{2}\right)^{\frac{3 n-m}{2 n}}\left(V_{1}\right)^{\frac{5 n-m}{4 n}}
\end{aligned}
$$

With Lemma 2, it follows from (27) that $y^{k} \rightarrow y^{k *}, \bar{x}^{k} \rightarrow \bar{x}^{k *}$, and $\omega_{i} \rightarrow \omega_{i}^{*}$ in a fixed time bounded by $T_{\max }^{1}$ as in (15).

The following results show the convergence of (13) to the ideal point of each objective.

Lemma 5: With the algorithm in (13), $\hat{x}_{i}^{k}$ for all $i \in \mathcal{V}, k=$ $1, \ldots, K$ converge to the ideal decision $\hat{x}_{i}^{k *}$ of each objective function $C_{i}^{k}$ in a fixed time, bounded by

$$
\begin{aligned}
T_{\max }^{2}= & \frac{1}{\gamma \underline{\kappa}^{\frac{m}{n}} 2^{\frac{m+n}{2 n}}\left(1-\frac{m+n}{2 n}\right)} \\
& +\frac{1}{\delta \underline{\kappa}^{2-\frac{m}{n}} 2^{\frac{3 n-m}{2 n}} K^{\frac{n-m}{2 n}}\left(\frac{3 n-m}{2 n}-1\right)} .
\end{aligned}
$$

Furthermore, $\hat{x}_{i}^{k}(t)$ for all $i \in \mathcal{V}, k=1, \ldots, K$ are bounded.

Proof: Define a Lyapunov candidate for agent $i$ as

$$
V_{2}=\frac{1}{2} \sum_{k=1}^{K}\left(\hat{x}_{i}^{k}-\hat{x}_{i}^{k *}\right)^{2}
$$

of which the time derivative along (13) is given by

$$
\begin{aligned}
\dot{V}_{2} & =\sum_{k=1}^{K}\left(\hat{x}_{i}^{k}-\hat{x}_{i}^{k *}\right) \dot{\hat{x}}_{i}^{k} \\
& =\sum_{k=1}^{K}\left(\hat{x}_{i}^{k}-\hat{x}_{i}^{k *}\right)\left[-\gamma\left[\nabla C_{i}^{k}\left(\hat{x}_{i}^{k}\right)\right]^{\frac{m}{n}}-\delta\left[\nabla C_{i}^{k}\left(\hat{x}_{i}^{k}\right)\right]^{2-\frac{m}{n}}\right] \\
& \leq-\gamma \underline{\kappa}^{\frac{m}{n}} \sum_{k=1}^{K}\left(\hat{x}_{i}^{k}-\hat{x}_{i}^{k *}\right)^{\frac{m+n}{n}}-\delta \underline{\kappa}^{2-\frac{m}{n}} \sum_{k=1}^{K}\left(\hat{x}_{i}^{k}-\hat{x}_{i}^{k *}\right)^{\frac{3 n-m}{n}} \\
& \leq-\gamma \underline{\kappa}^{\frac{m}{n}} 2^{\frac{m+n}{2 n}} V_{2}^{\frac{m+n}{2 n}}-\delta \underline{\kappa}^{2-\frac{m}{n}} 2^{\frac{3 n-m}{2 n}} K^{\frac{n-m}{2 n}} V_{2}^{\frac{3 n-m}{2 n}} .
\end{aligned}
$$

With Lemma 2, it is straightforward to obtain that $\hat{x}_{i}$ converges to the ideal decision variables $\hat{x}_{i}^{*}$ within a fixed time $T_{\max }^{2}$, given by (28). Moreover, $\hat{x}_{j}^{k}(t)$ is governed by the dynamics in (13), with bounded $\nabla C_{i}^{k}$. It converges to the finite ideal point $\hat{x}_{i}^{k *}$ within $t<T_{\max }^{2}$ and thus $\hat{x}_{i}^{k}(t)$ is bounded for $t \geq 0$.

The following theorem reveals the overall convergence of the proposed algorithms for the MRAP.

Theorem 1: Under Assumptions 1 and 2, the algorithms (12)-(14) solve the MRAP (11).

Proof: From Lemmas 4 and 5, it follows that $\nabla u_{i}\left(x_{i}, \hat{x}_{i}, \omega_{i}\right)$ is bounded. Therefore, $x_{i}, \lambda_{i}$, and $z_{i}$ are all bounded. The rest 
of this proof is based on $t \geq \max \left\{T_{\max }^{1}, T_{\max }^{2}\right\}$, where $\bar{x}=$ $\bar{x}^{*}, \omega_{i}=\omega_{i}^{*}$, and $\hat{x}=\hat{x}^{*}$ are used. Note that the solution of (11) is unique as shown in Lemma 3. To ease convergence analysis, the compact form of the distributed algorithm is written as

$$
\begin{aligned}
& \dot{x}=-\nabla U\left(x, \hat{x}^{*}, \omega^{*}\right)-\lambda \\
& \dot{\lambda}=-\mathcal{L} \lambda-\mathcal{L} z+x-d \\
& \dot{z}=\mathcal{L} \lambda
\end{aligned}
$$

where $\nabla U\left(x, \hat{x}^{*}, \omega^{*}\right)=\left[\nabla u_{1}\left(x_{1}, \hat{x}_{1}^{*}, \omega_{1}^{*}\right), \ldots, \nabla u_{N}\left(x_{N}, \hat{x}_{N}^{*}\right.\right.$, $\left.\left.\omega_{N}^{*}\right)\right]^{T} \in \mathbb{R}^{N}, \lambda=\left[\lambda_{1}, \ldots, \lambda_{N}\right]^{T} \in \mathbb{R}^{N}, z=\left[z_{1}, \ldots, z_{N}\right]^{T} \in$ $\mathbb{R}^{N}$, and $d=\left[d_{1}, \ldots d_{N}\right]^{T} \in \mathbb{R}^{N}$.

The equilibrium point of (31), $\left(x^{*}, \lambda^{*}, z^{*}\right)$, can be obtained from

$$
\begin{aligned}
& \mathbf{0}=-\nabla U\left(x^{*}, \hat{x}^{*}, \omega^{*}\right)-\lambda^{*} \\
& \mathbf{0}=-\mathcal{L} \lambda^{*}-\mathcal{L} z^{*}+x^{*}-d \\
& \mathbf{0}=\mathcal{L} \lambda^{*}
\end{aligned}
$$

as

$$
\begin{aligned}
\nabla U\left(x^{*}, \hat{x}^{*}, \omega^{*}\right) & =-\lambda^{*} \\
\mathcal{L} z^{*} & =x^{*}-d \\
\mathcal{L} \lambda^{*} & =\mathbf{0} .
\end{aligned}
$$

A Lyapunov function is proposed as

$$
V_{3}=\frac{1}{2}\left\|x-x^{*}\right\|^{2}+\frac{1}{2}\left\|\lambda-\lambda^{*}\right\|^{2}+\frac{1}{2}\left\|z-z^{*}\right\|^{2}
$$

and its time derivative is

$$
\begin{aligned}
\dot{V}_{3}= & \left(x-x^{*}\right)^{T} \dot{x}+\left(\lambda-\lambda^{*}\right)^{T} \dot{\lambda}+\left(z-z^{*}\right)^{T} \dot{z} \\
= & \left(x-x^{*}\right)^{T}\left[-\nabla U\left(x, \hat{x}^{*}, \omega^{*}\right)-\lambda\right] \\
& +\left(\lambda-\lambda^{*}\right)^{T}(-\mathcal{L} \lambda-\mathcal{L} z+x-d)+\left(z-z^{*}\right)^{T} \mathcal{L} \lambda .
\end{aligned}
$$

Applying the results in (33)-(35) leads to

$$
\begin{aligned}
\dot{V}_{3}= & -\left(x-x^{*}\right)^{T}\left[\nabla U\left(x, \hat{x}^{*}, \omega^{*}\right)-\nabla U\left(x^{*}, \hat{x}^{*}, \omega^{*}\right)\right] \\
& -\left(x-x^{*}\right)^{T}\left(\lambda-\lambda^{*}\right) \\
& -\left(\lambda-\lambda^{*}\right)^{T} \mathcal{L}\left(\lambda-\lambda^{*}\right)-\left(\lambda-\lambda^{*}\right) \mathcal{L}\left(z-z^{*}\right) \\
& +\left(\lambda-\lambda^{*}\right)^{T}\left(x-x^{*}\right)+\left(z-z^{*}\right)^{T} \mathcal{L}\left(\lambda-\lambda^{*}\right) \\
= & -\left(x-x^{*}\right)^{T}\left[\nabla U\left(x, \hat{x}^{*}, \omega^{*}\right)-\nabla U\left(x^{*}, \hat{x}^{*}, \omega^{*}\right)\right] \\
& -\left(\lambda-\lambda^{*}\right)^{T} \mathcal{L}\left(\lambda-\lambda^{*}\right) .
\end{aligned}
$$

Since the graph is connected, we have $\mathcal{L}$ is positive semidefinite, yielding

$$
-\left(\lambda-\lambda^{*}\right)^{T} \mathcal{L}\left(\lambda-\lambda^{*}\right) \leq 0 .
$$

Notice that

$$
\bar{u}_{i}\left(C_{i}^{k}\left(x_{i}\right)\right)=\sum_{j=1}^{K}\left[\omega_{i}^{k}\left(C_{i}^{k}\left(x_{i}\right)-C_{i}^{k}\left(\hat{x}_{i}^{*}\right)\right)^{p}\right]^{\frac{1}{p}} \forall 1 \leq p<\infty
$$

is a strictly convex and increasing function on $C_{i}^{k}\left(x_{i}\right) \geq$ $C_{i}^{k}\left(\hat{x}_{i}^{*}\right)$. Also, all the objective functions $C_{i}^{k}\left(x_{i}\right)$ are strongly convex on $x_{i}$. Thus, $U\left(x, \hat{x}^{*}, \omega^{*}\right)$ is strictly convex on $x$. Consequently

$$
-\left(x-x^{*}\right)^{T}\left[\nabla U\left(x, \hat{x}^{*}, \omega^{*}\right)-\nabla U\left(x^{*}, \hat{x}^{*}, \omega^{*}\right)\right] \leq 0 .
$$

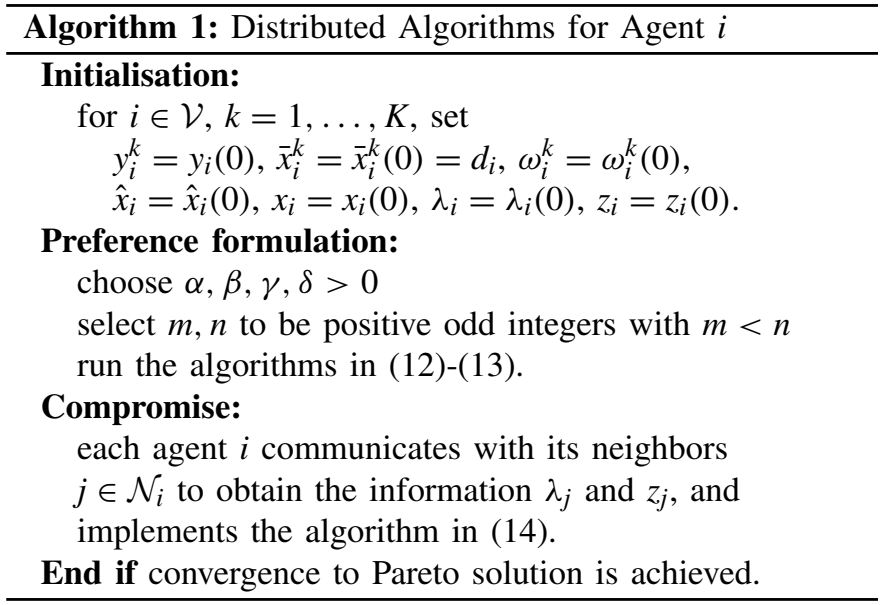

Then, it is clear that $\dot{V}_{3} \leq 0$ and $\dot{V}_{3}=0$ if and only if the equilibrium point is achieved. This completes the proof.

For real-time implementations, the overall structure of the proposed algorithms for each agent is summarized in Algorithm 1.

Remark 7: The proposed algorithms encompass fixed-time processes in (12) and (13), converging to the optimal solution of each type of objective summation $\sum_{i=1}^{N} C_{i}^{k}\left(\bar{x}_{i}^{k *}\right)$, and the ideal point of every local objective $C_{i}\left(\hat{x}_{i}^{*}\right)$, respectively. Then, the weights $\omega_{i}^{*}$ and the reference points $C_{i}\left(\hat{x}_{i}^{*}\right)$ are obtained for the decision makers. Therefore, the decision makers can learn from the optimization process in order to select a better solution from the Pareto-optimal set. The rest of algorithms in (14) employs gradient descent dynamics that compromise among the objectives, and search for the decision vectors $x_{i}$ mostly preferred by the decision makers. Similar algorithms for single-objective optimizations are studied in some recent works, e.g., [9], [31], and [32].

Remark 8: There is no global coordinator required in the proposed algorithms. It can be understood in the way that all local decision makers within the agents cooperatively optimize the multiobjective functions subject to the global constraint and specified preferences, by communication with their adjacent neighbors. The local information required for agent $i$ include the preference $u_{i}$ and cost functions $C_{i}$, which are regarded as private information in this article. The shared information from its neighbors are $y_{j}, \nabla C_{j}\left(\bar{x}_{j}\right), \lambda_{j}$, and $z_{j}$ for $j \in \mathcal{N}_{i}$. Therefore, the proposed algorithms are fully distributed.

Remark 9: In comparison to centralized methods, the distributed algorithms developed in this article can be employed with limited processing power and communication resources. From the algorithms in (12)-(14), it is clear that each agent only needs to communicate with its adjacent neighbors and, therefore, the communication complexity is independent of the total number of agents in the network. Meanwhile, the computation complexity is determined by the number of neighboring agents, since it only processes information received from the neighbors $j \in \mathcal{N}_{i}$. Moreover, it has been proved that the proposed algorithms converge to the unique Pareto solution satisfying the decision makers' preferences in a 


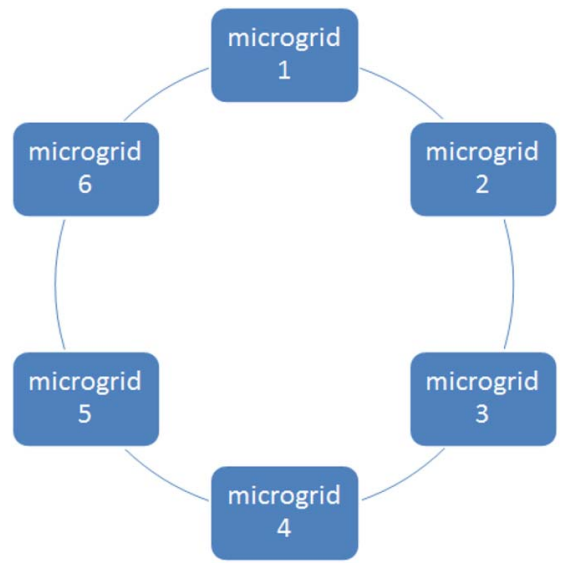

Fig. 4. Communication graph of the network with six agents.

deterministic sense. In Lemmas 4 and 5, fixed-time convergences to the optimal preference indices are achieved, and in Theorem 1, asymptotic convergence to the compromised solution is established via Lyapunov theories.

\section{Simulation ExAmPLES}

In this section, we apply the proposed algorithms to a group of six agents for algorithm validation, and then 120 agents for the scalability test, respectively. Weighted $L_{2}$ preference indices are used for the simulation, and the communication network is assumed to be represented by a circle graph as shown in Fig. 4 for the case with six agents, and a similar structure is utilized for the 120 agents. The objectives of each microgrid are formulated as

$$
\begin{aligned}
\min _{P_{d, i} \in \mathbb{R}} & \left\{C_{\text {eco }, i}\left(P_{d, i}\right), C_{\text {env }, i}\left(P_{d, i}\right), C_{\mathrm{tec}, i}\left(P_{d, i}\right)\right\} \\
\text { subject to } & \sum_{i=1}^{N} P_{d, i}=\sum_{i=1}^{N}\left(1+\eta_{i}\right) P_{l, i}
\end{aligned}
$$

where $C_{\mathrm{eco}, i}\left(P_{d, i}\right)=a_{d, i} P_{d, i}^{2}+b_{d, i} P_{d, i}+c_{d, i}, \quad C_{e n v, i}\left(P_{d, i}\right)=$ $r_{t}\left(a_{\mathrm{fuel}, i} P_{d, i}^{2}+b_{\text {fuel }, i} P_{d, i}+c_{\text {fuel }, i}\right)$, and $C_{\text {tec }, i}\left(P_{d, i}\right)=a_{\text {tec }, i}\left(P_{d, i}-\right.$ $\left.P_{\mathrm{opt}, i}\right)^{2}$ denote the economic, environmental, and technical objectives, respectively.

\section{A. Algorithm Validation and Comparison With Centralized Methods}

The simulation parameters are displayed in Table I, taken from the practical application in [33] with modifications, and $r_{t}=0.2$. Simulation results of power outputs are shown in Fig. 5(a), where the dotted lines represent the optimal solutions. It can be observed that the compromised Pareto solution is obtained very fast, which is due to the implementation of the fixed-time algorithms. Fig. 5(b) demonstrates that the power supply and demand are balanced, that is, the resource constraints are satisfied in the optimal states. In the simulation study, there is no central authority required to coordinate the distributed subsystems. All the agents only need to compromise their local cost functions, and measure their own resource. By exchanging some nonconfidential information, including $y_{i}, \lambda_{i}$, and $z_{i}$, with their adjacent neighbors, all the agents can cooperatively optimize the network objectives, and meanwhile meet the global resource constraints. As shown in Fig. 6, the weighting factors $\omega$ converge to some optimal values determined by the fixed-time algorithms, which are then used to form the preferences of the agents.

For comparison purposes, we implement the centralized primal-dual algorithms in [34] for the problem as well. Both of the algorithms share a similar structure, but the centralized method assumes that the Lagrangian multipliers are globally available to all the agents in the network. In particular, all the computational burdens are taken by one central unit to process the data collected from the agents, which include the private cost functions and preferences. Those are critical drawbacks of the centralized algorithms. When the participants are distributed over a large network, the centralized approaches may no longer be feasible due to communication and computation costs. Fig. 7(a) shows the optimal power outputs of the agents, and Fig. 7(b) depicts the supply-demand profile, where the constraints of network resource are satisfied. Comparing the results obtained from the distributed and centralized algorithms, it can be observed that both of them converge to the same optimal solution, which convinces that the distributed formulation is equivalent to the centralized one, as discussed in Remark 5. The convergence speed of the distributed algorithms is faster than the centralized ones, due to the implementation of fixed-time algorithms used in the preference formulation.

\section{B. Online Formulation of the Preferences}

For different settings of cost functions and constraints, the proposed algorithms are able to automatically adjust the performance index such that they can be implemented in an online manner. To show that we consider time-varying load profiles as shown in Fig. 8 displaying the weekly energy consumptions of a typical area in the U.K. (available at https://data.gov.uk/dataset). In each 30-min period, all the parameters in Table I are assumed to be randomly generated with variations in the range of $[-20 \%, 20 \%]$, and $r_{t}$ is randomly selected from $[0.15,0.3]$. Fig. 9(a) and (b) presents, respectively, the power outputs and the supply demand profiles of the network. Fig. 10 illustrates the changes of the weighting factors over one day. It is clear that each agent amends its preference index to achieve better tradeoff according to the importance of each objective. As the load varies, the cost of the objective will change, and therefore the weights are modified. This is an important property to achieve fully autonomous operations for power output regulations. Note that the communication and computation complexity of each agent is independent of the size of the network, and therefore the distributed algorithms can be implemented for large-scale networks. In comparison, centralized methods are not feasible since the communication and computation burden will be overwhelming as the number of participants increases.

\section{Scalability Test}

Distributed algorithms have attracted significant attention from the researchers in recent years, mainly due to their great scalability for large networks. In this section, the proposed 
TABLE I

PARAMETERS OF THE MiCROGRIDS

\begin{tabular}{lcccccccccc}
\hline & & \multicolumn{4}{c}{ economic objectives } & \multicolumn{3}{c}{ environmental objectives } & \multicolumn{2}{c}{ technical objectives } \\
& $P_{l, i}(\mathrm{~kW})$ & $\eta_{i}$ & $a_{d, i}$ & $b_{d, i}$ & $c_{d, i}$ & $a_{f u e l, i}$ & $b_{f u e l, i}$ & $c_{f u e l, i}$ & $a_{\text {tec }, i}$ & $P_{\text {opt }, i}(\mathrm{~kW})$ \\
\hline microgrid 1 & 120 & 0.023 & 0.086 & 3.482 & 3.481 & 0.175 & 1.266 & 0.666 & 1.000 & 90 \\
microgrid 2 & 150 & 0.054 & 0.093 & 4.688 & 4.263 & 0.165 & 1.665 & 3.171 & 1.088 & 120 \\
microgrid 3 & 114 & 0.032 & 0.072 & 2.533 & 3.500 & 0.117 & 1.359 & 1.308 & 1.336 & 135 \\
microgrid 4 & 150 & 0.048 & 0.080 & 2.300 & 6.578 & 0.120 & 1.323 & 2.973 & 0.788 & 90 \\
microgrid 5 & 186 & 0.050 & 0.098 & 4.210 & 4.810 & 0.206 & 1.937 & 1.487 & 1.220 & 90 \\
microgrid 6 & 90 & 0.056 & 0.090 & 2.312 & 1.261 & 0.156 & 1.173 & 2.073 & 1.140 & 75 \\
\hline
\end{tabular}

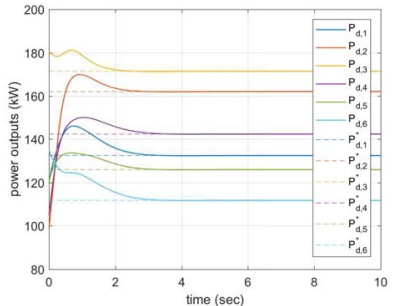

(a)

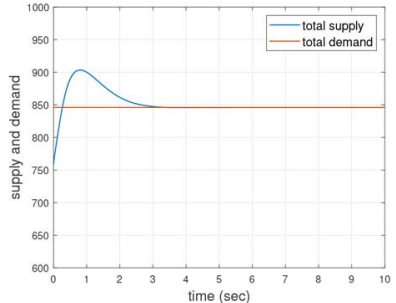

(b)
Fig. 5. Simulation results using the distributed algorithms. (a) Power outputs of the microgrids. (b) Supply and demand profiles.

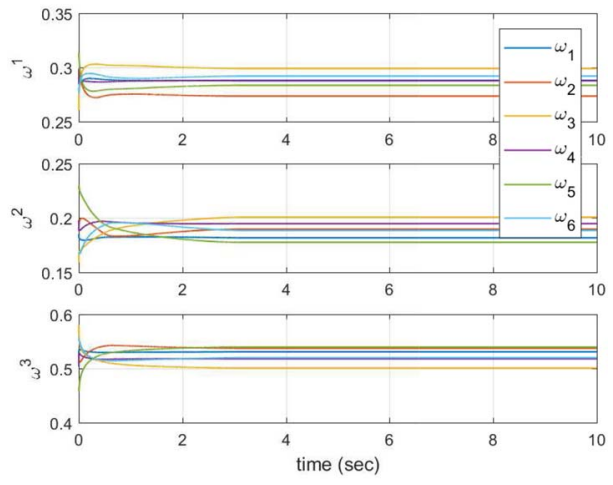

Fig. 6. Weighting factors of the objectives using the distributed algorithms.

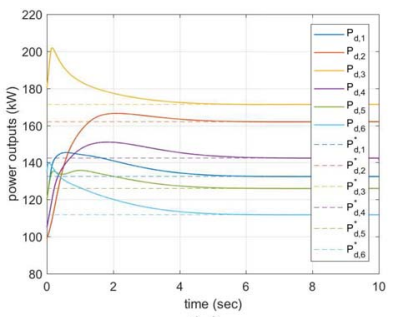

(a)

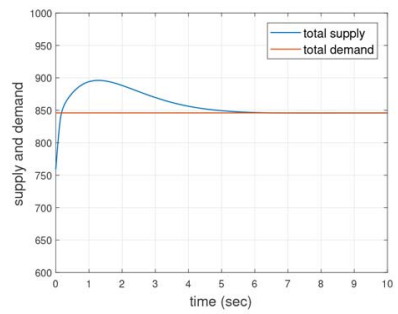

(b)
Fig. 7. Simulation results using the centralized algorithms. (a) Power outputs of the microgrids. (b) Supply and demand profiles.

algorithms will be implemented for a network of 120 agents. The parameters of the agents are randomly generated from Table I within the range of $\pm 30 \%$, and the communication topology is assumed to be a cycle. The optimal power outputs are displayed in Fig. 11(a). The convergence speed reduces slightly due to the increase of the network size, but it can be adjusted by changing the gains in the algorithms (12)-(14) if required. In Fig. 11(b), the optimal resource allocation is achieved, where the power supply and demand are balanced.

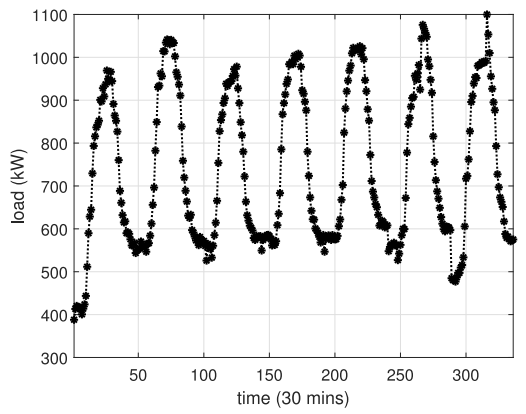

Fig. 8. Weekly power consumptions with a resolution of $30 \mathrm{mins} /$ period.

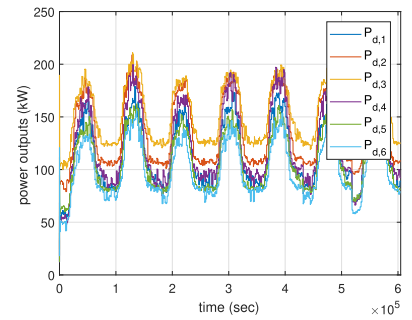

(a)



(b)
Fig. 9. Simulation results using the distributed algorithms with online preference formulation. (a) Power outputs of the microgrids. (b) Supply and demand profiles.

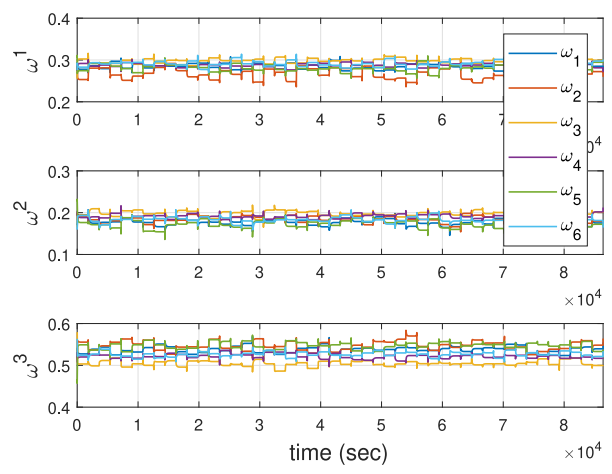

Fig. 10. Weighting factors $\omega$ for online preference formulation.

As having been discussed in Remark 9, the computation complexity of the algorithms is independent of the total number of participants in the network. Because each agent has two adjacent neighbors in a cycle graph, the communication requirement for each participant is the same as the previous case in Section IV-A. This also means that the computation complexity is limited to processing data from the agent itself 


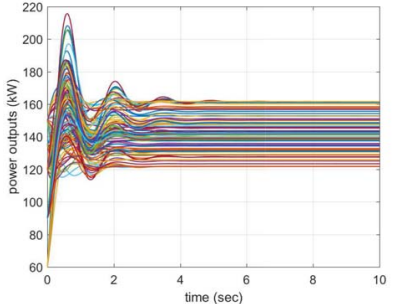

(a)

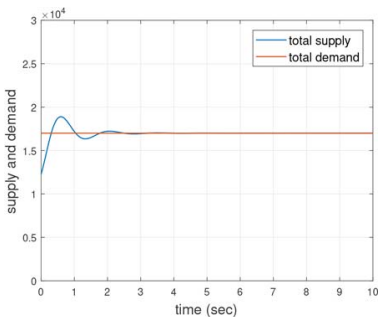

(b)
Fig. 11. Simulation results using the distributed algorithms with 120 agents. (a) Power outputs of the microgrids. (b) Supply and demand profiles.

and its two neighbors. Therefore, the proposed algorithms are feasible to be deployed in large networks.

\section{CONCLUSION}

This article has considered distributed multiobjective optimization for resource allocation problems over network connected multiagent systems. A preference-based method has been used to develop the most satisfactory Pareto solution, where the weighted $L_{p}$ metrics obtained by online calculation are implemented to reach robust solutions. Indeed, through two fixed-time optimization algorithms, ideal values and weighting factors are obtained accordingly. The proposed algorithms are fully distributed, which do not require the exchanging of private information. More important, the distributed methods can be implemented for large-scale distributed multiagent systems with potentially less communication and computation requirements. It has been proved that, under any connected graphs, the distributed algorithms converge to the Pareto solution in a deterministic manner. The simulation studies have demonstrated the effectiveness of the proposed algorithms.

\section{REFERENCES}

[1] Q. Liu, S. Yang, and J. Wang, "A collective neurodynamic approach to distributed constrained optimization," IEEE Trans. Neural Netw. Learn. Syst., vol. 28, no. 8, pp. 1747-1758, Aug. 2017.

[2] B. Gharesifard and J. Cortés, "Distributed continuous-time convex optimization on weight-balanced digraphs," IEEE Trans. Autom. Control, vol. 59, no. 3, pp. 781-786, Mar. 2014.

[3] Z. Li, Z. Ding, J. Sun, and Z. Li, "Distributed adaptive convex optimization on directed graphs via continuous-time algorithms," IEEE Trans. Autom. Control, vol. 63, no. 5, pp. 1434-1441, May 2018.

[4] A. Nedic, A. Ozdaglar, and P. A. Parrilo, "Constrained consensus and optimization in multi-agent networks," IEEE Trans. Autom. Control, vol. 55, no. 4, pp. 922-938, Apr. 2010.

[5] T. Zhao and Z. Ding, "Distributed agent consensus-based optimal resource management for microgrids," IEEE Trans. Sustain. Energy, vol. 9, no. 1, pp. 443-452, Jan. 2018.

[6] K. Senel and M. Akar, "A power allocation algorithm for multi-tier cellular networks with heterogeneous QoS and imperfect channel considerations," IEEE Trans. Wireless Commun., vol. 16, no. 11, pp. 7184-7194, Nov. 2017.

[7] G.-R. Iordanidou, C. Roncoli, I. Papamichail, and M. Papageorgiou, "Feedback-based mainstream traffic flow control for multiple bottlenecks on motorways," IEEE Trans. Intell. Transp. Syst., vol. 16, no. 2, pp. 610-621, Apr. 2015.

[8] Z. Deng, S. Liang, and Y. Hong, "Distributed continuous-time algorithms for resource allocation problems over weight-balanced digraphs," IEEE Trans. Cybern., vol. 48, no. 11, pp. 3116-3125, Nov. 2018.
[9] S. S. Kia, "Distributed optimal in-network resource allocation algorithm design via a control theoretic approach," Syst. Control Lett., vol. 107, pp. 49-57, Sep. 2017.

[10] P. Yi, Y. Hong, and F. Liu, "Initialization-free distributed algorithms for optimal resource allocation with feasibility constraints and application to economic dispatch of power systems," Automatica, vol. 74, pp. 259-269, Dec. 2016.

[11] H. Ren, W. Zhou, K. Nakagami, W. Gao, and Q. Wu, "Multi-objective optimization for the operation of distributed energy systems considering economic and environmental aspects," Appl. Energy, vol. 87, no. 12, pp. 3642-3651, 2010.

[12] R. Yang and L. Wang, "Multi-objective optimization for decision-making of energy and comfort management in building automation and control," Sustain. Cities Soc., vol. 2, no. 1, pp. 1-7, 2012.

[13] B. Tang, Z. Zhu, H.-S. Shin, A. Tsourdos, and J. Luo, "A framework for multi-objective optimisation based on a new self-adaptive particle swarm optimisation algorithm," Inf. Sci., vol. 420, pp. 364-385, Dec. 2017.

[14] K. Deb, Multi-Objective Optimization Using Evolutionary Algorithms. Hoboken, NJ, USA: Wiley, 2005.

[15] H. Xu, W. Zeng, X. Zeng, and G. G. Yen, "An evolutionary algorithm based on Minkowski distance for many-objective optimization," IEEE Trans. Cybern., vol. 49, no. 11, pp. 3968-3979, Nov. 2019.

[16] X. Cai, Z. Mei, Z. Fan, and Q. Zhang, "A constrained decomposition approach with grids for evolutionary multiobjective optimization," IEEE Trans. Evol. Comput., vol. 22, no. 4, pp. 564-577, Aug. 2018.

[17] J. Chen, J. Li, and B. Xin, "DMOEA- $\varepsilon$ C: Decomposition-based multiobjective evolutionary algorithm with the $\varepsilon$-constraint framework," IEEE Trans. Evol. Comput., vol. 21, no. 5, pp. 714-730, Oct. 2017.

[18] L. Chen, H.-L. Liu, K. C. Tan, Y.-M. Cheung, and Y. Wang, "Evolutionary many-objective algorithm using decomposition-based dominance relationship," IEEE Trans. Cybern., vol. 49, no. 12, pp. 4129-4139, Dec. 2019.

[19] A. Nedic and J. Liu, "Distributed optimization for control," Annu. Rev. Control, Robot. Auton. Syst., vol. 1, pp. 77-103, May 2018.

[20] S. Yang, Q. Liu, and J. Wang, "A collaborative neurodynamic approach to multiple-objective distributed optimization," IEEE Trans. Neural Netw. Learn. Syst., vol. 29, no. 4, pp. 981-992, Apr. 2018.

[21] J. Chen and A. H. Sayed, "Distributed Pareto optimization via diffusion strategies," IEEE J. Sel. Topics Signal Process., vol. 7, no. 2, pp. 205-220, Apr. 2013.

[22] R. Wang, Q. Zhang, and T. Zhang, "Decomposition-based algorithms using Pareto adaptive scalarizing methods," IEEE Trans. Evol. Comput., vol. 20 , no. 6 , pp. 821-837, Dec. 2016.

[23] K. Miettinen, Nonlinear Multiobjective Optimization. New York, NY, USA: Springer, 1998.

[24] Z. Ding, "Consensus control of a class of Lipschitz nonlinear systems," Int. J. Control, vol. 87, no. 11, pp. 2372-2382, 2014.

[25] Z. Zuo, B. Tian, M. Defoort, and Z. Ding, "Fixed-time consensus tracking for multiagent systems with high-order integrator dynamics," IEEE Trans. Autom. Control, vol. 63, no. 2, pp. 563-570, Feb. 2018.

[26] A. Polyakov, "Nonlinear feedback design for fixed-time stabilization of linear control systems," IEEE Trans. Autom. Control, vol. 57, no. 8, pp. 2106-2110, Aug. 2012.

[27] J. Jahn, Vector Optimization. Berlin, Germany: Springer, 2009.

[28] P. L. Yu, "A class of solutions for group decision problems," Manag. Sci., vol. 19, no. 8, pp. 936-946, 1973.

[29] Y. Sawaragi, H. Nakayama, and T. Tanino, Theory of Multiobjective Optimization. Orlando, FL, USA: Academic, 1985.

[30] P. L. Yu, Multiple-Criteria Decision Making: Concepts, Techniques, and Extensions, vol. 30. New York, NY, USA: Springer, 1985.

[31] T. Zhao and Z. Ding, "Distributed finite-time optimal resource management for microgrids based on multi-agent framework," IEEE Trans. Ind. Electron., vol. 65, no. 8, pp. 6571-6580, Aug. 2018.

[32] G. Chen and Z. Li, "A fixed-time convergent algorithm for distributed convex optimization in multi-agent systems," Automatica, vol. 95, pp. 539-543, Sep. 2018.

[33] T. Zhao, Z. Li, and Z. Ding, "Consensus-based distributed optimal energy management with less communication in a microgrid," IEEE Trans. Ind. Inf., vol. 15, no. 6, pp. 3356-3367, Jun. 2019.

[34] D. P. Bertsekas, Constrained Optimization and Lagrange Multiplier Methods. Boston, MA, USA: Academic, 1982. 


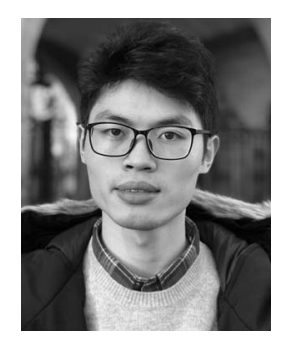

Zhongguo Li (Student Member, IEEE) received the first B.Eng. degree in electrical and electronic engineering from the University of Manchester, Manchester, U.K., and the second B.Eng. degree in communication engineering from Jilin University, Jilin, China, in 2017. He is currently pursuing the $\mathrm{Ph} . \mathrm{D}$. degree in electrical and electronic engineering with the University of Manchester.

His research interests include distributed optimization and control multiagent systems and their applications.

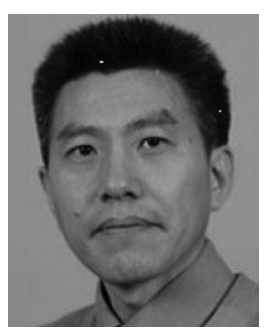

Zhengtao Ding (Senior Member, IEEE) received the B.Eng. degree from Tsinghua University, Beijing, China, in 1984, and the M.Sc. degree in systems and control and the Ph.D. degree in control systems from the University of Manchester Institute of Science and Technology, Manchester, U.K., in 1986 and 1989, respectively.

He was a Lecturer with Ngee Ann Polytechnic, Singapore, for ten years. In 2003, he joined the University of Manchester, Manchester, where he is the Professor of Control Systems with the Department of Electrical and Electronic Engineering. He has authored the book Nonlinear and Adaptive Control Systems (IET, 2013) and a number of journal papers. His research interests include nonlinear and adaptive control theory and their applications.

Prof. Ding has served as an Associate Editor for the IEEE TRANSACTIONS on Automatic Control, Transactions of the Institute of Measurement and Control, Control Theory and Technology, Mathematical Problems in Engineering, Unmanned Systems, and the International Journal of Automation and Computing. 